\title{
A tradução cinematográfica de Il Gattopardo, de Giuseppe Tomasi di Lampedusa
}

\author{
Marinês Lima Cardoso ${ }^{1}$ \\ Universidade do Estado do Rio de Janeiro \\ marinesrj@yahoo.com.br
}

RESUMO: Este trabalho busca traçar uma análise relacionada à tradução cinematográfica do romance Il Gattopardo, de Giuseppe Tomasi di Lampedusa e o filme homônimo dirigido por Luchino Visconti, tomando como base o narrador, o espaço e o tempo. Esses elementos narratológicos são importantes na articulação e no desenvolvimento do material narrativo tanto no cinema quanto na literatura. Entretanto, são percebidos de modo diferente nas referidas obras. Romance e filme retratam as reflexões do protagonista diante da unificação italiana e dos seus desdobramentos, como a substituição de classes sociais, a saber, o declínio da aristocracia e a ascensão da burguesia. Apesar de se aproximar da obra de partida ao reproduzir as situações, os diálogos e o ambiente em que as cenas se verificam, Visconti apresenta uma outra leitura da obra na conclusão, ao dar enfoque maior a um capítulo do livro e ao excluir outros do filme. Esse tipo de tradução é, assim, uma intervenção, em que se evidencia determinado tipo de interpretação através de uma releitura do texto literário.

Palavras-chave: Literatura Italiana. Tradução Cinematográfica. Il Gattopardo.

ABSTRACT: The goal of this paper is to analyze the adaptation of the novel Il Gattopardo, by Giuseppe Tomasi Di Lampedusa into the film of the same title directed by Luchino Visconti, in regard to the narrator, space and time. These narrative elements are important in articulating and developing the material in film and literature alike; however, these are perceived differently in each piece of work. The novel and the film depict the main character's thoughts in the context of the Italian unification and its outcomes, such as the displacement of social classes, the decline of the aristocracy and the rise of the bourgeoisie. Despite its proximity with the original work with the reproduction of situations, pieces of dialogue and the places in which the scenes take place, Visconti presents a different point of view on the novel in the film's conclusion, focusing more on a specific chapter of the book whilst removing others from the film. This type of adaptation is, as a matter of fact, an intervention, in which a certain type of interpretation is evidenced through a rereading of the literary text.

\footnotetext{
${ }^{1}$ Doutora em Letras Neolatinas pela UFRJ. Atualmente, é Professora Adjunta de Língua, Literatura e Cultura italianas da UERJ.
} 
Keywords: Italian literature. Film translation. Il Gattopardo

$\mathcal{A}$ relação entre cinema e literatura sempre despertou um grande debate entre críticos das duas áreas artísticas. No início, a união entre eles não era evidente, pois, quando surgiu, o cinema não tinha um caráter narrativo. Quando os irmãos Lumière exibiram Saída dos Operários da Fábrica, em 1895, oficialmente a primeira sessão de cinema no mundo, eles não vislumbravam o casamento que se daria no século seguinte entre o cinema e a literatura. Nos primeiros anos de seu nascimento, o cinema poderia ser apenas um instrumento de investigação científica, um documentário ou uma simples diversão, pois fora concebido como um meio de registro. Ele não tinha, ainda, a vocação de contar histórias.

Segundo Aumont et al (2013, p. 89), existem algumas razões básicas para a união entre essas duas formas de arte. Uma delas seria o estatuto da imagem em movimento, que, em perpétua transformação, mostra a passagem de um estado de coisa representada para um outro estado. É uma transformação da imagem em movimento através do tempo. Qualquer objeto ou paisagem, mesmo estático, sofrerá essa transformação pelo simples fato de ser filmado, ou seja, no cinema, o representado é sempre um devir. A outra razão seria mais um fato histórico, pois trata-se da "busca da legitimidade" do cinema. Nos seus primórdios, o cinema não desfrutava do prestígio das "artesnobres", como o teatro e o romance, e, para sair dessa condição de um espetáculo um tanto vil, passou a organizar o seu material através de uma narrativa.

Angelo Moscariello (1981, p. 03) indica o caráter narrativo do filme, em que as noções de narratividade são fundamentais para um estudo mais profundo, ou seja, embora sejam representações autônomas e independentes, o destino narrativo é também comum ao cinema, fato que o libera da ideia de uma arte somente visiva. 
A partir desse elemento comum entre o cinema e a literatura, muitas obras literárias começaram a ser adaptadas para as telas do cinema. O termo "adaptação" é utilizado há muito tempo nos estudos tradicionais como sinônimo de modificação de um texto de um sistema de linguagem para outro. Na nova visão, a adaptação passa a ser considerada uma instância da tradução, ou seja, é um fenômeno da tradução, vista, principalmente, como uma diferença entre os dois textos, e não como semelhança.

A transposição para o cinema é definida por Betton como:

... uma recriação: o tradutor (...) realiza uma obra pessoal e manifesta-se não como 'ilustrador', mas como um verdadeiro criador: inspira-se numa obra literária - todo artista extrai sua argila, sua matéria bruta, do patrimônio cultural (1987, p. 112).

A dificuldade de transmitir a mesma mensagem, a do texto literário, através de um sistema de linguagem diferente, o cinema, é compartilhada por muitos críticos. A questão da fidelidade em relação ao texto de partida se fez presente nos estudos iniciais entre literatura e cinema, pois alguns críticos defendiam esse princípio no processo de adaptação. Betton (1987, p. 115) afirma que a fidelidade ao texto de partida é muito rara, senão impossível, pois não se pode representar visualmente significados verbais, assim como é impossível exprimir com palavras o que está representado em formas e cores. Além disso, ainda segundo o autor, a imagem que surge da leitura de um romance é fundamentalmente diferente da do filme, pois esta imagem é baseada em um dado real que é oferecido para se ver, e não para se imaginar, como ocorre no romance. Atualmente, a transposição e a busca por equivalentes entre os diferentes suportes sígnicos já caracterizam um processo de tradução. Desse modo, as adaptações cinematográficas podem ser consideradas traduções, uma vez que sofrem as mesmas imposições que qualquer tradução.

Neste trabalho, buscar-se-á tratar alguns aspectos relacionados à tradução cinematográfica de um romance, a saber, Il Gattopardo, de Giuseppe 
Tomasi di Lampedusa, publicado em 1958, e o filme homônimo, sob a direção de Luchino Visconti, produzido em 1963, tomando como base alguns elementos da narrativa, como o narrador, o espaço e o tempo. O romance de Lampedusa constituiu um verdadeiro "caso" literário italiano na época de sua publicação, uma vez que obteve um grande sucesso junto ao público, mas despertou muitas discussões entre aqueles que o consideravam um grande romance da narrativa contemporânea e outros que o viam como um "frutto fuori stagione", que se limitava a uma perspectiva decadente ou reacionária. De fato, seu escritor fora severamente criticado devido à temática trazida pelo romance naqueles anos, ou seja, um episódio já superado pela sociedade italiana e pelos escritores na história italiana, aquele da unificação italiana e os seus desdobramentos.

Il Gattopardo narra a história do príncipe don Fabrizio e os seus questionamentos sobre a mudança que se verifica na Sicília durantes os anos da Unificação do Estado italiano. $\mathrm{Na}$ verdade, trata-se de uma análise do protagonista sobre a decadência da aristocracia siciliana, ou melhor, o declínio de um mundo do qual ele era um forte representante. O personagem observa com um distanciamento irônico e melancólico as transformações políticas e sociais que se verificam ao seu redor, pois sabe que nada pode impedir esse novo quadro que se configura na segunda metade do século XIX.

A obra de Visconti também obteve sucesso de público e rendeu o prêmio Palma de Ouro no Festival de Cannes, em 1963. Vale destacar que o diretor também era herdeiro da aristocracia, como era o caso de Lampedusa:

Nel ritratto del protagonista, il principe di Salina, Visconti finisce per identificarsi fino in fondo, scavando dentro se stesso e andando ad individuare i suoi timori e le sue illusioni, ma anche evidenziando la coscienza storicistica" (GIOIA, 2014, p. 112).

Assim, através dos temas do romance de Lampedusa, o diretor italiano representa a decadência de uma família aristocrática, de uma classe social e de uma inteira época e a instauração de uma nova sociedade, a burguesia, na qual 
triunfam a desordem e a mediocridade que substituem a aristocrática harmonia do passado.

É interessante destacar que Visconti se aproxima bastante do romance pelos diálogos dos personagens, pelas situações e pela decoração do ambiente em que circulam os personagens. Segundo Gioia (2014, p. 114), o filme foi muito preciso na cenografia, através de longas pesquisas sobre a arquitetura do barroco siciliano, bem como na decoração dos ambientes, utilizando, frequentemente, móveis da época. Para o diretor, eram fundamentais o rigor da reconstrução e o refinamento e a autenticidade da decoração para aderir à obra de Lampedusa, pois não se pode esquecer que o romance é a história de uma passagem de poderes, do fim de uma classe social dominante que via o refinamento e a elegância como elementos indispensáveis à sua existência. Por isso, era necessário ao diretor descrever essa elegância particular dessa classe social.

Esse processo metamórfico de um signo para outro que se verifica nas obras em estudo é definido por Plaza da seguinte maneira: "é aquele tipo de tradução que consiste na interpretação dos signos verbais por meio de sistemas de signos não verbais" (2010, p. XI). Observa-se, assim, que, para o autor, a passagem de um sistema verbal para um não verbal constitui um processo de tradução, existindo dois signos, o traduzido, que é a obra literária, e o signo tradutor, que é a tradução para o cinema.

A primeira definição sobre esse tipo de tradução, a intersemiótica, foi feita por Jakobson, através da sua famosa divisão:

Distinguimos três maneiras de interpretar um signo verbal: ele pode ser traduzido em outros signos da mesma língua, em outra língua, ou em outro sistema de símbolos não-verbais. Essas três espécies de tradução devem ser diferentemente classificadas: 1) A tradução intralingual, ou reformulação, consiste na interpretação dos signos verbais por meio de outros signos da mesma língua; 2) A tradução interlingual, ou tradução propriamente dita, consiste na interpretação dos signos verbais por meio de alguma outra língua; 3) A tradução intersemiótica, ou transmutação, consiste na interpretação dos signos verbais por meio de sistemas de signos não-verbais (1995, p. 62). 
Essa definição também ecoa em Umberto Eco, que aponta que a tradução intersemiótica ocorre nos casos em que:

não se traduz de uma língua natural para outra, mas entre sistemas semióticos diversos entre si, como quando, por exemplo, se "traduz" um romance para um filme, um poema épico para uma obra em quadrinhos ou se extrai um quadro do tema de uma poesia" (2010, p. 09).

Para o ensaísta, esse tipo de tradução é também uma interpretação, pois várias concessões são feitas quando se elabora um filme partindo de um romance. As escolhas estéticas e as apropriações por parte do diretor serão, assim, inevitáveis e necessárias. Observa-se, dessa maneira, que todo ato de traduzir é um processo de recriação, de expansão de significados. Essa recriação pressupõe alargar as possibilidades de leitura da obra literária e não somente trazer para a tela uma narrativa pré-existente.

Como tratado anteriormente, o romance em estudo traz à cena uma família aristocrática que assiste ao fim de uma era, a da monarquia dos Bourbons, e à anexação do reino da Sicília ao novo governo do rei Vittorio Emanuele. A obra de Lampedusa narra, assim, a história de uma grande mudança política e social que, no arco de uma década, vê a Itália se transformar em uma nação, unificando a maior parte do seu território. É oportuno destacar que o centro do romance não são os acontecimentos históricos e sociais, mas as reflexões do protagonista, que revelam um estado de cansaço e de impotência diante da realidade e a constatação de caminhar em direção ao fim, ou seja, à morte. Baldi et al. ilustram bem esse aspecto:

... Il Gattopardo è in realtà un romanzo psicologico incentrato sullo scavo interiore del protagonista, il principe Salina, sul suo senso di stanchezza e di impotenza di fronte alle trasformazioni in atto, sulla sua percezione dell'incombere della morte" (2011, p. 252).

Além da extinção da sua classe social, don Fabrizio está ciente de que a morte física também se aproxima. Várias são as indicações dessa percepção no romance e no filme. A cena de contemplação do protagonista diante de um quadro que retrata a morte de um homem em seu leito, reunido pelos 
familiares evoca reflexões no protagonista. Ele imagina sua esposa e filhas chorando diante da sua morte já eminente. Percebe-se, assim, que para o príncipe é como se dois mundos estivessem em um processo de conclusão, o da sua classe e a sua própria vida. Do mesmo modo que a aristocracia deve ceder espaço a uma nova classe social que surge, ele deve ceder espaço a uma nova geração que substituirá a sua.

Tancredi, seu sobrinho, representa a figura que encarnará esse novo papel na sociedade, pois, embora de origem nobre, soubera se adequar e participar ativamente dos novos rumos que as transformações trouxeram e tenta convencer seu tio sobre isso, com a famosa frase: "Se vogliamo che tutto rimanga come è, bisogna che tutto cambi" (LAMPEDUSA, 1982, p. 21). Ou seja, haverá uma substituição de classe sociais, mas a sociedade se reconstituirá idêntica a si mesma, na mesma divisão entre ricos e pobres, fortes e fracos, patrões e empregados. Don Fabrizio, representante de uma aristocracia estática e incapaz de se adaptar às mudanças sócio-políticas, não quer seguilas, entretanto, consente o matrimônio entre Tancredi e Angelica, filha de don Calogero. Apesar de não concordar com o sobrinho, existe entre ambos um profundo afeto. Tancredi vê no tio um modelo de classe, de grandiosidade e de refinamento, enquanto don Fabrizio se espelha no jovem, projetando nele as suas aspirações, a sua vontade de viver e de amar.

Ao mesmo tempo em que se verifica o declínio da velha aristocracia, ocorre a ascensão da burguesia, representada pelo prefeito da cidade, Don Calogero. Este soubera prosperar financeiramente, mas não tinha o prestígio das famílias aristocráticas. Além de ter acumulado uma fortuna quase igual à da família Salina, apresenta uma forte influência política que lhe permitirá ser deputado em Turim. Segundo Gioia (1982, p.119), essa substituição de classes representa, por um lado, uma promoção social, pois a burguesia, constituída de ex-camponeses, tornar-se-á a classe dominante e, por outro, uma degradação político e moral. Esse aspecto pode ser verificado no romance, na 
descrição do fraque que don Calogero usa na ocasião do jantar oferecido na mansão da família Salina: “... si poteva affermare che, come riuscita sartoriale, il frack di Don Calogero era una catastrofe. Il panno era finissimo, il modello recente, ma il taglio era semplicemente mostruoso" (LAMPEDUSA, 1982, p. 52). Don Fabrizio observa com pessimismo essa substituição de classes, pois os novos patrões são incultos e não têm o bom gosto e a classe dos antigos. A esse propósito, Gioia afirma que: "Cambieranno le apparenze, per lui e quelli come lui, quell'aristocrazia che ha il monopolio dell'intelligenza e del buongusto, che d'altronde sono la giustificazione della sua esistenza” (2014, p. 26). É justamente essa intuição de deterioração das formas que imprime, no príncipe e no ambiente, uma melancolia e um pessimismo.

O declínio dessa classe social se verifica através de um lento processo de fusão entre a aristocracia e a burguesia, sendo percebido pelo protagonista em vários momentos. Tancredi faz o papel de interlocutor entre esses dois grupos, buscando se integrar a esse novo mundo, pois se abre a possibilidade de uma brilhante carreira no novo reino unitário. O seu casamento com Angelica sela a aliança de interesses com as novas classes em ascensão.

Convém destacar que todo texto narrativo, seja literário, seja audiovisual, possui algumas estruturas comuns e é através do confronto entre essas estruturas e aquelas do texto literário de partida que esse tipo de tradução pode ser estudado. Coutinho afirma que o cinema sofre várias influências da literatura,

... de logo manifestando-se a da continuidade da novela ou do romance tradicionais, o que descobre a arraigada dependência do filme em relação ao enredo, à história, que de direito pertence à literatura; tal herança viuse facilitada pelo tipo de apresentação de dois gêneros artísticos: ambos se processam no tempo, segundo a índole sucessiva das recepções (1996, p.104).

A obra literária possui um narrador que relata os fatos ao leitor, enquanto no filme, as imagens, em uma quantidade infinita, adquirem uma instância narrativa. No cinema, é a câmera que exerce a função narrativa, pois 
focaliza, recorta e aproxima as imagens para o espectador. Convém destacar que essa câmera que tudo registra não é neutra, existe alguém que seleciona e combina as imagens que serão apresentadas ao público. Conforme esclarecem Aumont et al., na narrativa cinematográfica, o narrador seria

o diretor, na medida em que ele escolhe determinado tipo de encadeamento narrativo, determinado tipo de decupagem, determinado tipo de montagem, por oposição a outras possibilidades oferecidas pela linguagem cinematográfica (2013, p. 111).

Dessa maneira, o narrador é aquele que produz, simultaneamente, uma narrativa e uma história.

No romance em destaque, existe um narrador em terceira pessoa que informa ao leitor os fatos, mas o ponto de vista é o do príncipe Salina, pois tudo é filtrado através do seu olhar e das suas reflexões. É através dessas reflexões que se pode verificar, por exemplo, a presença constante da morte no romance, como a recordação da imagem de um soldado morto encontrado na sua propriedade. Para Gioia (2014, p. 32), essa percepção do fim que se aproxima representa a parábola da sua vida que está se concluindo, uma vez que o príncipe já desempenhou todas as suas obrigações familiares e sociais. Ele constata que a estirpe da sua família chegou ao fim da história e que representa o último descendente da família Salina. O narrador o descreve como um homem forte e alto:

Non che fosse grasso: era soltanto immenso e fortissimo; la sua testa sfiorava (nelle case abitate dai comuni mortali) il rosone inferiore dei lampadari; le sue dita potevano accartocciare come carta velina le monete da un ducato (LAMPEDUSA, 1982, p.06).

Na obra de Visconti, o aspecto físico do protagonista e o domínio exercido sobre toda a família já são evidenciados na abertura. Todos estão reunidos e ajoelhados na sala, rezando o rosário com o padre Pirrone, no momento em que um falatório interrompe essa atividade diária da família. A câmera capta a influência forte do protagonista sobre a família quando ele, 
repentinamente, fecha a bíblia, mostrando, assim, que a oração se concluíra naquele momento. Nas cenas seguintes, todos esperam uma posição e observam passivamente a movimentação do príncipe diante das novas notícias que chegam naquele momento, como a descoberta do cadáver de um soldado no jardim e a carta de um parente informando a don Fabrizio que as tropas de Garibaldi desembarcaram em Palermo. Todos se curvam e aceitam as decisões tomadas pelo patriarca da família.

Em outra cena, destaca-se o receio do padre por ter aborrecido o príncipe, após criticá-lo por aceitar o que estava acontecendo na Itália. O padre não vê de modo positivo a nova situação que começa a se instaurar na sociedade, pois a próxima ordem a ser instituída será comandada por pessoas que não valorizam a tradição e só pensam nos bens materiais. Visconti se aproxima da obra de Lampedusa ao reproduzir o mesmo discurso do príncipe em que explica ao padre a sua visão diante do que está acontecendo:

Non siamo ciechi, caro Padre, siamo soltanto uomini. Viviamo in una realtà mobile alla quale cerchiamo di adattarci come le alghe si piegano sotto la spinta del mare. Alla Santa Chiesa è stata esplicitamente promessa l'immortalità; a noi, in quanto classe sociale, no (LAMPEDUSA, 1982, p. 29).

Segundo o protagonista, a igreja deve mesmo se preocupar com os acontecimentos sociais e políticos em curso, uma vez que não está destinada à morte, enquanto a aristocracia tenta se adequar ao que está acontecendo para não desaparecer nesse momento de mudança e sobreviver por mais um período.

Ainda a propósito do narrador, vale também ressaltar a descrição de Angelica na entrada da mansão da família Salina:

Era alta e benfatta, in base a generosi criteri; la carnagione sua doveva possedere il sapore della crema fresca alla quale rassomigliava, la bocca infantile quello delle fragole. Sotto la massa dei capelli color di notte avvolti in soavi ondulazioni, gli occhi verdi albeggiavano, immoti come quelli delle estatue e, com'essi, un po' crudeli. Procedeva lenta, facendo roteare intorno a sé l'ampia gonna bianca e recava nella persona la 
pacatezza, l'invincibilità della donna di sicura bellezza (LAMPEDUSA, 1982, p. 52-53).

Após a chegada desastrosa de seu pai, a jovem entra triunfalmente na mansão, encantando a todos com a sua beleza. Segundo Gioia (2014, p. 52), ela, representante da jovem classe social que surge, apresenta uma beleza animalesca, pois lembra um animal cheio de vitalidade e saúde. Convém destacar que Angelica é plenamente consciente da força da sua beleza que a eleva sobre todas as outras mulheres.

Visconti também soube retratar a estreia exuberante de Angelica na mansão da família Salina, enfocando a sua entrada lenta e direcionando os olhares dos demais personagens sobre a jovem. A câmera se aproxima sobre seu rosto e, em seguida, enquadra o seu corpo. Observa-se, desse modo, que o diretor, através de imagens em movimento, buscou traduzir a descrição física do personagem feita por Lampedusa.

Além do narrador, o espaço e o tempo constituem elementos importantes na articulação e no desenvolvimento do material narrativo tanto no cinema quanto na literatura. Entretanto, esses elementos são percebidos de modo diferente nas obras narrativa e fílmica. Pellegrini et al. afirmam que todas as formas narrativas, sejam as literárias, sejam as visuais:

\footnotetext{
...estão direta ou indiretamente articuladas em sequências temporais, não importa se lineares, se truncadas, invertidas ou interpoladas. A diferença entre a literatura e o cinema, nesse caso, é que, na primeira, as sequências se fazem com palavras e, no segundo, com imagens (2003, p.18).
}

Enquanto na literatura, a questão do tempo pode ser descrita com precisão ao longo da leitura, como, por exemplo, quando se indica, em poucas palavras, que se passaram dias, meses ou anos, no cinema, essa percepção não é tão precisa, embora a sensação do tempo fluindo se verifique através das imagens em movimento. No romance em estudo, os capítulos se iniciam com o mês e o ano dos acontecimentos, permitindo, desse modo, situar 
temporalmente o leitor durante a leitura. O leitor sabe exatamente quando se inicia a história, maio de 1860, e quando esta termina, maio de 1910.

Outras marcações temporais podem ainda ser observadas no romance, como a duração da viagem que a família Salina faz em direção a Donnafugata: "Il viaggio era durato tre giorni ed era stato orrendo" (LAMPEDUSA, 1982, p. 39). Toda a família e os empregados se deslocam pelas estradas quentes para passarem as férias de verão na pequena cidade em que a família Salina era reverenciada pelos habitantes.

$\mathrm{Na}$ narrativa fílmica, o espectador toma conhecimento do ano em que é ambientada a história através dos acontecimentos históricos, no caso, a unificação italiana entre os anos 1860 e 1861. A percepção da passagem temporal se verifica através do espaço que muda, como, por exemplo, quando se mostra a família dormindo em uma pousada de estrada durante o percurso para Donnafugata. A câmera enfoca as paredes sujas do cômodo em que os membros da família repousam, mostrando o desconforto de todos e a decadência que já se instaura. Conforme o espaço muda, percebe-se que o tempo avançou, revelando, assim, que o tempo no filme é, em geral, relativo a um espaço. Isso porque, no filme, tempo e espaço são inseparáveis, pois as relações temporais adquirem um aspecto quase espacial do mesmo modo que o espaço adquire características temporais.

Enquanto o aspecto temporal, no filme, está relacionado ao espaço, a representação deste último é mais perceptível do que no romance, uma vez que na obra literária são necessárias muitas palavras para indicar uma imagem que no filme é percebida em um instante. No cinema, a espacialização do elemento temporal produz profundas alterações nas formas de perceber o espaço e de representá-lo. A esse propósito, Hauser afirma que:

O espaço perde a qualidade estática, a passividade serena, e torna-se agora dinâmico; adquire existência diante de nossos olhos, por assim dizer. É fluido, ilimitado, inacabado, um elemento com história própria, com seu próprio esquema e processo de desenvolvimento" (2003, p. 971). 
É por isso que Pellegrini et al. utilizam os termos invisível e visível para se referirem, respectivamente, ao tempo e ao espaço: “... o tempo, que é invisível, é preenchido com o espaço ocupado por uma sequência de imagens visíveis; misturam-se, assim, o visível e o invisível" (2003, p.18).

Gaudreault e Jost, no estudo sobre a narrativa cinematográfica, pontuam que o espaço apresenta uma primazia sobre o tempo, devido ao caráter imagético do filme. Ou seja, o espaço antecede o tempo:

E, já que o fotograma vem antes da sucessão de fotogramas, a temporalidade no cinema deve efetivamente se apoiar sobre o espaço para chegar a se inscrever no centro da narrativa. O tempo não começa a existir a não ser quando se opera a passagem entre um primeiro fotograma (que já é espaço) e um segundo (que também já é espaço) (2009, p. 105).

Enquanto isso, o narrador da narrativa escritural deve fazer uma espécie de discriminação, uma vez que ele não pode descrever, ao mesmo tempo, o quadro espacial e a ação no qual esta se passa. Desse modo, ele deve escolher o que narrar, ocorrendo, muitas vezes, a ausência de algumas informações espaciais consideradas "decorativas" e não úteis à narrativa. Devido à sua capacidade de exprimir tudo, a língua não poderá nunca representar todas as relações de ordem espacial e, por isso, uma narrativa escritural não conseguirá retratar simultaneamente os parâmetros espaciais e temporais com os quais ela se relaciona. Em Il Gattopardo, a paisagem é frequentemente descrita, revelando a sua relação com história:

Il giardino dormiva sprofondato nell'ombra, sotto; nell'aria inerte gli alberi sembravano di piombo fuso; dal campanile incombente giungeva il sibillo fiabesco dei gufi. Il ciero era sgombro di nuvole; quelle che avevano salutato a sera se ne erano andate chissà dove (...) Le stelle apparivano torbide e i loro raggi faticavano a penetrare la coltre di afa. (LAMPEDUSA, 1982, p. 57)

Já na narrativa fílmica, o espaço está sempre presente, pois as informações relativas ao aspecto espacial são fornecidas em abundância pela 
câmera. No cinema, há a apresentação simultânea de vários elementos informacionais e não somente uma determinada informação espacial. No filme de Visconti, é interessante destacar que o espaço representa um elemento importante, pois a natureza é capturada em diversas ambientações. $\mathrm{Na}$ cena em que Tancredi se despede da família e parte na sua carruagem para os combates, todos correm em direção à sacada do terraço e contemplam a partida do jovem pela vasta propriedade da família. Nessa mesma cena, observa-se uma fusão entre os personagens estáticos e a paisagem, como se fossem pinturas de um quadro.

Observa-se, desse modo, que para o cinema é muito difícil dar o enfoque apenas na ação, e não no quadro espacial no qual esta se desenrola. Além disso, no filme, o espaço natural adquire uma importância vital ao relacionar seu caráter imutável à Sicilia. Na cena em que o príncipe e don Ciccio saem para caçar nas primeiras horas do dia, o espectador visualiza os aspectos geográficos do pequeno vilarejo, em que se revela um aspecto selvagem e áspero do campo. O espectador toma conhecimento desse espaço que se mostra através do deslocamento dos dois homens pelas terras áridas e improdutivas. É nesse lugar que don Fabrizio colhe informações do que se passara em Donnafugata nos últimos meses e, ao mesmo tempo, a natureza se apresenta diante dos olhos dos espectadores.

Como já fora evidenciado antes, a morte é um elemento que transparece nas duas narrativas. Entretanto, Visconti decide excluir do filme o capítulo que narra a morte do protagonista e dar um espaço maior ao baile oferecido pela família Ponteleone. Enquanto o romance se conclui com as irmãs Salina já envelhecidas, o diretor conclui o filme com o baile, que ocupa um terço do filme. A propósito da dilatação do tempo que ocupa essa festa, o próprio diretor explica:

La verità è che quella scena era necessaria alla struttura interna del film proprio nella sua apparente sproporzione. Sono 40 minuti che ne rappresentano infinitamente di più (...) Il principe, in quei 40 minuti, 
No livro, o baile ocupa o sexto capítulo e o leitor é informado da data da sua realização, novembro de 1862. Já no capítulo seguinte, a história avança em vinte anos e vem descrita a morte do príncipe de Salina em um quarto de hotel. Para o diretor que optou por retirar do filme a morte do protagonista, era necessário imprimir a esse momento o transcorrer do tempo para o príncipe durante essa festa. $O$ espectador assiste às cenas em que $O$ protagonista sente, inicialmente, um desconforto e cansaço e, progressivamente, esse desconforto se transforma em melancolia. A câmera destaca um grande contraste entre os jovens que se divertem e parecem ignorantes em relação ao que está por acontecer e um indivíduo exausto fisicamente que observa a todos com um olhar de compaixão. O príncipe vaga solitário pelos salões do palácio e os sinais físicos do seu cansaço são evidenciados pelas cenas em que ele frequentemente enxuga o rosto com um lenço e fala a um conhecido que não se sente bem. A relação com a morte fica mais evidenciada quando o personagem se dirige à biblioteca e observa atentamente a pintura La morte del Giusto, di Greuze. Nesse momento, ele se vê naquele quadro como se estivesse diante de um espelho, contemplando, assim, a própria morte. Como afirma o próprio diretor, era necessário transmitir ao espectador a percepção de que o tempo passara para o príncipe e que o seu declínio começava.

Tanto no romance quanto no filme, pode-se estabelecer uma relação entre a conclusão do baile e o declínio de uma era. Lampedusa descreve o fim do baile de modo muito preciso: "I volti delle signore erano lividi, gli abiti sgualciti, gli aliti pesanti (...) Nella sala del buffet, vuota, vi erano soltanto piatti smantellati bicchieri con un dito di vino...” (LAMPEDUSA, 1982, p. 160). Visconti também tece essa descrição através da câmera que circula pelo ambiente, permitindo vislumbrar o dia amanhecendo e os convidados, cansados e suados, despedindo-se dos anfitriões em um espaço que, 
inicialmente, marcado pelo luxo e pela abundância, agora está em total desordem, com pratos e copos vazios e espalhados sobre as mesas. Além disso, o diretor acrescenta um outro elemento que nos permite vislumbrar a saída de cena da aristocracia para dar espaço à nova burguesia. Trata-se do diálogo entre Angelica e Concetta, filha do príncipe, em que esta diz que está cansada de frequentar tantos bailes, enquanto aquela diz se sentir feliz nas festas oferecidas. Ou seja, a classe aristocrática se despede e cede lugar à outra que se instaurará. O baile, desse modo, pode ser lido como metáfora da conclusão de uma fase, a da aristocracia, e o início de outra, a da burguesia.

É durante esse baile que Angelica é apresentada à aristocracia como noiva de Tancredi. A participação da jovem é o coroamento da interessada história de amor com Tancredi e, ao mesmo tempo, o triunfo da nova classe dirigente: “...Angelica che invita Don Fabrizio a farla danzar è la borghesia che seduce l'aristocrazia un attimo prima di prenderne il posto..." (ANILE; GIANNICE, 2013, p. 209). O príncipe, desde a entrada de Angelica em sua casa, sentira-se seduzido pela beleza e exuberância da jovem e dá a sua permissão para o casamento. Além de bela, Angelica tem outros atributos que a tornam a escolha certa como esposa, tem uma fortuna considerável e uma presença altiva, sendo, assim, a companheira ideal para um jovem ambicioso como Tancredi.

Após pedir a Tancredi que providenciasse uma carruagem para sua família, o príncipe retorna para casa a pé, diante de uma cidade que desperta. Mais uma vez, a morte também estará presente nos caminhos do protagonista, pois, enquanto faz seu percurso, surge um padre que está levando a extrema unção a um doente. A câmera enfoca o príncipe que se ajoelha, enquanto o cortejo fúnebre avança e, em seguida, após ter visto a dor e o sofrimento, levanta-se, observa os primeiros raios de sol que surgem e caminha entre as vielas, afastando-se, de costas, da câmera. 
Enquanto o filme termina com o retorno do príncipe à casa, no romance, no sétimo capítulo, vem descrita a sua morte, lentamente já anunciada em diversos momentos da história, em um quarto de hotel. Ele observa os filhos e os netos que cercam a sua cama e constata que nenhum deles se assemelha a ele, ou seja, naquele momento se extingue a sua estirpe, " $\mathrm{i}$ gattopardi”. Em seguida, a obra atravessa um arco de quase três décadas e tem a sua conclusão com as irmãs Salina buscando ainda conservar o prestígio da família através das relíquias religiosas que conseguiram reunir durante décadas. Lampedusa parece, assim, revelar um último resquício de vida de uma família tradicional que vivera seu apogeu e assistiu à sua substituição por uma outra classe social.

Verificou-se, assim, nesse estudo, que a tradução cinematográfica é uma intervenção em que ocorre uma transformação audiovisual de um texto literário, evidenciando determinado tipo de interpretação e, assim, apresentando uma releitura do texto literário. Como fora observado anteriormente, Visconti se aproximara bastante da obra de partida reproduzindo os diálogos, as situações e o ambiente em que as cenas se verificaram, entretanto, apresentou uma outra leitura na conclusão da sua obra ao retirar do filme alguns capítulos do livro e dar enfoque maior a um outro.

\section{Referências bibliográficas}

ANILE, Alberto; GIANNICE, Maria Gabriella. Operazione Gattopardo. Genova: Feltrinelli, 2013.

AUMONT et al. A estética do filme. Campinas: Papirus, 2013.

BALDI et al. Testi e storia della Letteratura. Dal dopoguerra ai giorni nostri. Vol. G. Torino: Paravia, 2011.

BETTON, Gérard. Estética do cinema. São Paulo: Martins Fontes, 1987. 
A tradução cinematográfica de /l Gattopardo, de Giuseppe Tomasi di Lampedusa Marinês Lima Cardoso

COUTINHO, Evaldo. A imagem autônoma. São Paulo: Editora Perspectiva, 1996.

ECO, Umberto. Quase a mesma coisa. Rio de Janeiro: BestBolso, 2011.

GAUDREAULT, Andre. JOST, François. A narrativa cinematográfica. Brasília: Editora Universidade de Brasília, 2009.

GIOIA, Edvige. Il mondo decadente delGattopardo. Roma: Aracneeditrice, 2014.

HAUSER, Arnold. História social da arte e da literatura. São Paulo: Martins Fontes, 2003.

LAMPEDUSA, Giuseppe. Il Gattopardo. Milano: Feltrinelli, 1982.

MOSCARIELLO, Angelo. Cinema e/o letteratura. Bologna: Pitagora Editrice, 1981.

PELLEGRINI, Tânia et al. Literatura, cinema e televisão. São Paulo: Ed. Senac: São Paulo, 2003.

PLAZA, Julio. Traducão Intersemiótica. São Paulo: Ed. Perspectiva, 2010.

\section{Referência filmográfica}

Il Gattopardo. Direção: Luchino Visconti. Produção: Goffredo Lombardo. Roteiro: Suso Cecchi D’Amico, Luchino Visconti, Pasquale Festa Campanile, Enrico Medioli. Versátil, 1963. 185 min, colorido. 


\section{La traduzione cinematografica de Il Gattopardo, di Giuseppe Tomasi di Lampedusa ${ }^{2}$}

Marinês Lima Cardoso 3

RIASSUNTO: Questo lavoro cerca di fare un'analisi riguardante la traduzione cinematografica del romanzo Il Gattopardo di Giuseppe Tomasi di Lampedusa e il film omonimo diretto da Lucchino Visconti, basandosi specificamente sul narratore, sul tempo e sullo spazio. Questi elementi narratologici sono importanti per l'articolazione e per lo sviluppo del materiale narrativo sia nel cinema che nella letteratura, ma vengono percepiti in modo diverso nelle due opere messe a confronto. Romanzo e film espongono le riflessioni del protagonista nei confronti dell'unificazione italiana e delle sue conseguenze, come, ad esempio, la sostituzione delle classi sociali, cioè il declino dell'aristocrazia e l'ascesa della borghesia. Malgrado s'avvicini all'opera di partenza, riproducendo le situazioni, i dialoghi e l'ambiente in cui le scene si svolgono, Visconti presenta un'altra lettura dell'opera alla conclusione del film, dando maggior rilievo a uno specifico capitolo del libro, escludendone altri. Questo tipo di traduzione risulta così come un intervento in cui viene evidenziata determinatasorta di interpretazione attraverso la rilettura del testo letterario.

Parole-Chiave: Letteratura Italiana. Traduzione intersemiotica cinematografica. Il Gattopardo.

ABSTRACT: The goal of this paper is to analyze the adaptation of the novel Il Gattopardo, by Giuseppe Tomasi Di Lampedusa into the film of the same title directed by Luchino Visconti, in regard to the narrator, space and time. These narrative elements are important in articulating and developing the material in film and literature alike; however, these are perceived differently in each piece of work. The novel and the film depict the main character's thoughts in the context of the Italian unification and its outcomes, such as the displacement of social classes, the decline of the aristocracy and the rise of the bourgeoisie. Despite its proximity with the original work with the reproduction of situations, pieces of dialogue and the places in which the

\footnotetext{
2 Versione di Alcebiades Arêas e Edvaldo Sampaio Belizário.

${ }^{3}$ Dottoressa in Lettere Neolatine presso la UFRJ. Attualmente, è docente di Lingua, Letteratura e Cultura italiane presso la UERJ
} 
scenes take place, Visconti presents a different point of view on the novel in the film's conclusion, focusing more on a specific chapter of the book whilst removing others from the film. This type of adaptation is, as a matter of fact, an intervention, in which a certain type of interpretation is evidenced through a rereading of the literary text.

Keywords: Italian literature. Film translation. Il Gattopardo

$\mathcal{L}$ a relazione tra cinema e letteratura ha sempre suscitato un grande dibattito tra i critici delle due aree artistiche. All'inizio, l'unione tra di esse non era evidente, poiché, al suo sorgere, il cinema non aveva un carattere narrativo. Quando i fratelli Lumière esibirono L'uscita dalle officine Lumière nel 1895, ufficialmente la prima proiezione cinematografica pubblica, essi non intravedevano il matrimonio che avrebbe avuto luogo nel ventesimo secolo tra cinema e letteratura. Nei primi anni della sua nascita, il cinema potrebbe essere solo uno strumento di indagine scientifica, un documentario o un semplice divertimento visto che era stato creato come uno strumento di registrazione. Esso non era destinato a raccontare storie.

Secondo Aumont et al. (2013, p. 89), ci sono delle ragioni fondamentali perché queste due forme di arte si uniscano. Una di esse sarebbe lo statuto dell'immagine in movimento che, in perpetua trasformazione, rivela il passaggio da uno stato di cose rappresentato ad un altro. È una trasformazione dell'immagine in movimento attraverso il tempo. Qualsiasi oggetto o paesaggio, anche se statico, subirà questa trasformazione semplicemente perché è stato filmato, ossia, nel cinema, il rappresentato è sempre un divenire. L'altra ragione sarebbe specialmente un fatto storico, giacché significa "la ricerca della legittimità" del cinema. Ai suoi albori, il cinema non godeva del prestigio delle "arti nobili", come per esempio il teatro e il romanzo; e, per uscire da questa condizione scomada, di disprezzo, cominciò ad organizzare il suo materiale attraverso una narrazione. 
Angelo Moscariello (1981, p. 03) indica il carattere narrativo del film in cui le nozioni di narratività risultano fondamentali per uno studio più approfondito, cioè, nonostante siano rappresentazioni autonome e indipendenti, il destino narrativo è anche comune al cinema, il che lo libera dall'idea di un'arte esclusivamente visiva.

A partir da quest'elemento comune, tra il cinema e la letteratura, parecchie opere letterarie sono state adattate per il cinema. Il termine "adattamento" viene utilizzato da molto tempo negli studi tradizionali come sinonimo di modificazione di un testo da un sistema di linguaggio all'altro. Nella nuova visione, l'adattamento diventa un'esigenza della traduzione, ossia, risulta un fenomeno della traduzione, visto, soprattutto, come una differenza fra i due testi e non come una somiglianza.

La trasposizione per il cinema viene definita da Betton come:

... una ricreazione: il traduttore (...) realizza un'opera personale e si manifesta non da 'illustratore', ma da vero creatore: s'ispira in un'opera letteraria - ogni artista estrae la sua argilla, la sua materia bruta, dal patrimonio culturale" (1987, p. 112).

La difficoltà di trasmettere lo stesso messaggio, quello del testo letterario, attraverso un sistema diverso di linguaggio come il cinema, viene condiviso da molti critici. La questione della fedeltà riguardo al testo di partenza si è fatta presente negli studi tra letteratura e cinema, dato che alcuni critici difendevano questo principio nel processo di adattamento. Betton (1987, p. 115) afferma che la fedeltà al testo di partenza è molto rara, o addirittura impossibile, dato che non si può rappresentare visualmente i significati verbali, così come è impossibile esprimere a parole quello che è raffigurato attraverso forme e colori. Inoltre, ancora secondo l'autore, l'immagine che sorge dalla lettura di un romanzo è fondamentalmente diversa da quella di un film, poiché quest'immagine è basata su un dato reale che viene offerto per vedere e non invece per immaginarsi come succede nel romanzo. Odiernamente, la trasposizione e la ricerca di equivalenti tra $i$ diversi supporti segnici 
caratterizzano già un processo di traduzione. Così, gli adattamenti cinematografici possono essere considerati traduzioni, visto che subiscono le stesse imposizioni fatte a qualunque altra traduzione.

In questo articolo tratteremo specificamente degli elementi: narratore, tempo e spazio nella traduzione cinematografica del romanzo Il Gattopardo, di Giuseppe Tomasi di Lampedusa, pubblicato nel 1958, in confronto al film omonimo, diretto da Lucchino Visconti, del 1963. Il romanzo di Lampedusa costituì un autentico "caso" letterario all'epoca della sua pubblicazione dato che riscosse un grande successo di pubblico, ma suscitò molte discussioni tra quelli che lo consideravano un grande romanzo della narrativa contemporanea e quegli altri che lo vedevano come un "frutto fuori stagione", limitato a una prospettiva decadente o reazionaria. Infatti, Lampedusa fu severamente criticato dovuto alla tematica dell'opera in quegli anni, vale a dire all'episodio dell'unificazione d'Italia e le sue conseguenze, già superato dalla società italiana e dagli scrittori nella storia italiana.

Ne Il Gattopardo si narra la storia del principe don Fabrizio e i suoi questionamenti sul cambiamento che si verifica in Sicilia durante gli anni dell'Unificazione d'Italia. In verità, si tratta di un'analisi del protagonista sulla decadenza dell'aristocrazia siciliana, o meglio, sul declino di un mondo del quale era stato un illustre esponente. Il personaggio osserva con un allontanamento ironico e malinconico le trasformazioni politiche e sociali che si verificano intorno a lui, perché sa che niente può impedire questo nuovo quadro che si configura nella seconda metà del XIX secolo.

Anche l'opera di Visconti ottenne successo di pubblico e vinse la Palma d'oro nel Festival di Cannes nel 1963. È degno di nota che il regista era anche erede dell'aristocrazia come era il caso di Lampedusa:

Nel ritratto del protagonista, il principe Salina, Visconti finisce per identificarsi fino in fondo, scavando dentro se stesso e andando a individuare $\mathrm{i}$ suoi timori e le sue illusioni, ma anche evidenziando la coscienza storicistica" (GIOIA, 2014, p. 112). 
Così, attraverso i temi del romanzo di Lampedusa, il regista italiano rappresenta la decadenza di una famiglia aristocratica, di una classe sociale e di tutta un'epoca e l'instaurazione di una nuova società, la borghesia, nella quale trionfano il disordine e la mediocrità che sostituiscono l'aristocratica armonia del passato.

È interessante osservare che Visconti s'avvicina molto al romanzo attraverso i dialoghi dei personaggi, i contesti in cui si sviluppano le azioni, la decorazione dell'ambiente in cui si muovono i personaggi. Per Gioia (2014, p. 114), il film fu molto preciso riguardo gli apparati scenografici attraverso profonde ricerche sull'architettura del barocco siciliano oltre la decorazione degli ambienti, utilizzando, con frequenza, i mobili originali dell'epoca. Per il regista, erano troppo importanti il rigore della ricostruzione e la raffinatezza e l'autenticità dell'arredamento per aderire all'opera di Lampedusa, giacché non si può dimenticare che il romanzo costituisce la storia di un passaggio di poteri istituiti, la fine di una classe sociale dominante che vedeva la raffinatezza e l'eleganza come elementi indispensabili alla sua esistenza. Perciò era necessario che il regista descrivesse la particolare eleganza di questa classe sociale.

Questo processo metamorfico da un segno all'altro, che si verifica nelle opere a confronto, viene definito da Plaza così: "è quel tipo di traduzione che interpreta i segni verbali per mezzo di sistemi di segni non verbali" (2010, p. XI). Così si osserva che per l'autore il passaggio da un sistema verbale all'altro non verbale costituisce un processo di traduzione in cui ci sono due segni: il tradotto, che è l'opera letteraria; e quel traduttore, che è la traduzione per il cinema.

La prima definizione su questo tipo di traduzione, l'intersemiotica, la diede Jakobson, attraverso la famosa divisione:

Noi distinguiamo tre modi di interpretazione di un segno linguistico, secondo che lo si traduca in altri segni della stessa lingua, in un'altra lingua, o in un sistema di simboli non linguistici. Queste tre forme di traduzione debbono essere designate in maniera diversa: 1) la traduzione endolinguistica o riformulazione consiste nell'interpretazione dei 
segni linguistici per mezzo di altri segni della stessa lingua; 2) la traduzione interlinguistica o traduzione propriamente detta consiste nell'interpretazione dei segni linguistici per mezzo di un'altra lingua; 3) la traduzione intersemiotica o trasmutazione consiste nell'interpretazione dei segni linguistici per mezzo di sistemi di segni non linguistici. (1995, p. 62)

Questa definizione è ripresa anche da Umberto Eco. Secondo lui, la traduzione intersemiotica accade nei casi in cui "... non si traduce da una lingua naturale a un'altra, ma tra sistemi semiotici diversi tra loro, come quando per esempio si "traduce" un romanzo in un film, un poema epico in un'opera a fumetti oppure si trae un quadro dal tema di una poesia" (2010, p. 09). Per il saggista questo tipo di traduzione è anche un'interpretazione, dato che troppe concessioni sono fatte quando si produce un film a partire da un romanzo. Le scelte estetiche e le appropriazioni dalla parte del regista saranno inevitabili e necessarie. Si osserva, in questo modo, che ogni singolo atto del tradurre implica un processo di ricreazione, di espansione di significati. Questa ricreazione richiede di ampliare le possibilità di lettura dell'opera letteraria e non solo portare sullo schermo una narrativa preesistente.

Come accennato prima, il romanzo in evidenza mette in scena una famiglia aristocratica che assiste alla fine di un'epoca, cioè quella della monarchia dei Borboni, e l'annessione del regno di Sicilia al nuovo governo del re Vittorio Emanuele. L'opera di Lampedusa racconta, quindi, la storia di un grande cambiamento politico e sociale che nell'arco di un decennio vede l'Italia trasformarsi in una nazione, unificando la maggior parte del suo territorio. È opportuno evidenziare che al centro del romanzo non vengono posti gli avvenimenti storici e sociali, bensì le ponderazioni del protagonista che rivelano un senso di stanchezza e di impotenza davanti alla realtà e alla constatazione di camminare verso la sua fine, ossia, verso la morte. Baldi et al. illustrano bene questo aspetto:

Il Gattopardo è in realtà un romanzo psicologico incentrato sullo scavo interiore del protagonista, il principe Salina, sul suo senso di stanchezza e 
di impotenza di fronte alle trasformazioni in atto, sulla sua percezione dell'incombere della morte" (2011, p. 252).

Oltre la scomparsa della sua classe sociale, don Fabrizio è anche consapevole dell'avvicinarsi della morte fisica. Diverse sono le indicazioni di queste percezione nel romanzo e nel film. La scena emblematica della contemplazione del protagonista di fronte a un quadro che raffigura la morte di un uomo sul suo letto, circondato dalla famiglia evoca delle riflessioni al protagonista. Egli immagina sua moglie e le sue figlie in lacrime a causa della sua morte ormai vicina. Si capisce allora che, per il principe, è come se due mondi stessero in un processo di conclusione: quello della sua classe e quello della sua propria vita. Così come l'aristocrazia deve cedere spazio a una nuova classe sociale che sta sorgendo, egli deve, quindi, dare spazio a una nuova generazione che sostituirà la sua.

Tancredi, suo nipote, rappresenta la figura che incarnerà questo nuovo ruolo nella società, poiché, nonostante le origini nobili, seppe adeguarsi e partecipare attivamente ai nuovi orientamenti che le trasformazioni portarono e cerca di convincere suo zio di ciò, con la famosa frase: " Se vogliamo che tutto rimanga come è, bisogna che tutto cambi" (LAMPEDUSA, 1982, p. 21). Cioè, ci sarà una sostituzione di classi sociali, ma la società verrà ricostituita in maniera identica alla precedente, con la stessa divisione tra ricchi e poveri, forti e deboli, padroni e impiegati. Don Fabrizio, rappresentante di una aristocrazia estatica e incapace di adattarsi ai cambiamenti sociopolitici, non vuole seguirli, ma approva il matrimonio fra Tancredi e Angelica, figlia di don Calogero. Malgrado non sia d'accordo con il nipote, tra di loro esiste un profondo affetto. Tancredi vede nello zio un modello di classe, di grandiosiotà e raffinatezza, mentre don Fabrizio si specchia nel giovane, proiettando su di lui le proprie aspirazioni, la propria volontà di vivere e d'amare.

Allo stesso tempo in cui viene verificato il declino della vecchia aristocrazia, accade l'ascensione della borghesia, rappresentata dal sindaco 
della città, don Calogero. Questi seppe prosperare finanziariamente, ma non aveva il prestigio delle famiglie aristocratiche. Oltre ad aver accumulato una fortuna quasi uguale a quella dei Salina, gode di una grande influenza politica che gli permetterà di diventare deputato a Torino. Secondo Gioia (1982, p. 19), questa sostituzione di classi rappresenta, da un lato, una promozione sociale, poiché la borghesia, costituita da ex contadini, diverrà i nuovi padroni e, dall'altro, un degrado politico e morale. Questo aspetto può essere verificato nel romanzo nella parte in cui viene descritto il frac indossato da don Calogero durante la cena nella villa dei Salina: "... si poteva affermare che, come riuscita sartoriale, il frack di don Calogero era una catastrofe. Il panno era finissimo, il modello recente, ma il taglio era semplicemente mostruoso" (LAMPEDUSA, 1982, p. 52). Don Fabrizio vede con pessimismo questa sostituzione di classi, poiché i nuovi padroni sono incolti e non hanno il buongusto e la finezza degli antichi. A questo proposito Gioia afferma che "Cambieranno le apparenze, per lui e quelli come lui, quell'aristocrazia che ha il monopolio dell'intelligenza e del buongusto, che d'altronde sono la giustificazione della sua esistenza" (2014, p. 26). È proprio questa intuizione di deterioramento delle forme che imprimono, sia nel principe che nell'ambiente, un'atmosfera di malinconia e di pessimismo.

Il declino di questa classe sociale si verifica attraverso il lento processo di fusione tra l'aristocrazia e la borghesia, e viene percepito dal principe in diverse occasioni. Tancredi fa il ruolo dellinterlocutore tra questi due ceti e cerca di integrarsi in questo nuovo mondo, visto che si apre la possibilità di una brillante carriera nel nuovo regno unitario. Il suo matrimonio con Angelica sigilla un'alleanza di interessi con le nuove classi in ascesa.

Occorre sottolineare che il testo narrativo, sia letterario che audiovisivo, possiede alcune strutture comuni ed è attraverso il confronto tra queste strutture e quelle del testo letterario di partenza che questo tipo di traduzione 


\section{A tradução cinematográfica de // Gattopardo, de Giuseppe Tomasi di Lampedusa Marinês Lima Cardoso}

può essere studiato. Coutinho afferma che il cinema subisce molte influenze dalla letteratura,

...a priori manifestandosi quella della continuità della novella o del romanzo tradizionali, il che rivela la dipendenza radicata del film rispetto alla trama, alla storia, che di diritto spetta alla letteratura; questa eredità è stata facilitata da un tipo di presentazione di due generi artistici: entrambi sono elaborati nel tempo, secondo la natura successiva delle recezioni (1996, p. 104).

L'opera letteraria possiede un narratore che racconta $i$ fatti ai lettori mentre nel film, le immagini, in una quantità infinita, acquisiscono un'istanza narrativa. Nel cinema, è la camera che eseguisce la funzione narrativa, poiché focalizza, ritaglia e avvicina le immagini allo spettatore. Ł̀ opportuno evidenziare che questa camera che tutto registra non è neutra, c'è qualcuno che sceglie e organizza le immagini che saranno presentate al pubblico. Come chiariscono Aumont et al. (2013, p. 111), nella narrativa cinematografica, il narratore sarebbe "... il regista, visto che lui definisce un certo tipo di incatenamento narrativo, determinato tipo di découpage, determinato tipo di montaggio, in contrapposizione ad altre possibilità offerte dal linguaggio cinematografico". Così, il narratore è quello che produce, contemporaneamente, una narrativa e una storia.

Nel romanzo in evidenza c'è um narratore in terza persona che informa al lettore i fatti. Ma il punto di vista narrativo è quello del personaggio Salina, poiché tutto viene filtrato attraverso il suo sguardo e le sue riflessioni. È a partire da queste riflessioni che si può verificare, per esempio, la presenza costante della morte nel romanzo, come il ricordo dell'immagine di un soldato morto, trovato nella sua proprietà. Secondo Gioia (2004, p. 32), questa percezione della fine che s'avvicina rappresenta la parabola della sua vita che si sta concludendo, dato che il principe aveva già adempiuto a tutti i suoi obblighi famigliari e sociali. Egli si rende conto che la stirpe della sua famiglia era arrivata alla fine della storia e che egli rappresenta l'ultimo discendente dei Salina. 
Il narratore descrive il protagonista come un uomo robusto e alto:

Non che fosse grasso: era soltanto immenso e fortissimo; la sua testa
sfiorava (nelle case abitate dai comuni mortali) il rosone inferiore dei
lampadari; le sue dita potevano accartocciare come carta velina le monete
da un ducato..." (LAMPEDUSA, 1982, p.06).

Nell'opera di Visconti, le fattezze del protagonista e il dominio esercitato su tutta la famiglia vengono già evidenziati nell'inizio del film. Sono tutti riuniti e inginocchiati in uno dei sontuosi saloni del palazzo Salina recitando il rosario con il prete Pirrone nel momento in cui un brusio interrompe questa attività quotidiana della famiglia. La camera cattura la forte influenza del protagonista sulla famiglia quando egli rapidamente chiude la Bibbia, mostrando così che la preghiera si concluse in quel momento. Nelle scene successive, tutti quanti aspettano una posizione e osservano passivamente la movimentazione del principe davanti alle nuove notizie che arrivano in quel momento, come la scoperta del cadavere di un soldato nel giardino e la lettera di un parente informando a don Fabrizio che le truppe di Garibaldi sbarcarono a Palermo. Tutti si inchinano e accettano le decisioni prese dal patriarca della famiglia.

In un'altra scena, si mette in evidenza il timore del padre Pirrone per aver dato fastidio al principe, dopo averlo criticato per accettare quello che stava succedendo in Italia. Il prete non vede di buon occhio la nuova situazione che comincia ad instaurarsi nella società, visto che il prossimo ordine istituito verrà comandato da persone che non valorizzano la tradizione e pensano solo ai beni materiali. Visconti s'avvicina all'opera di Lampedusa quando riproduce lo stesso discorso del principe nel quale egli spiega al prete la sua visione davanti a quello che sta succedendo:

Non siamo ciechi, caro Padre, siamo soltanto uomini. Viviamo in una realtà mobile alla quale cerchiamo di adattarci come le alghe si piegano sotto la spinta del mare. Alla Santa Chiesa è stata esplicitamente promessa l'immortalità; a noi, in quanto classe sociale, no" (LAMPEDUSA, 1982, p. 29). 
Secondo il protagonista, la Chiesa deve addirittura preoccuparsi degli avvenimenti sociali e politici in corso, dato che non è destinata alla morte, mentre l'aristocrazia deve cercar di adeguarsi a quel che sta succedendo per non scomparire in questo momento di cambiamento e sopravvivere ancora per un po'.

Vale anche osservare la descrizione di Angelica fatta dal narratore all'ingresso del palazzo della famiglia Salina:

Era alta e benfatta, in base a generosi criteri; la carnagione sua doveva possedere il sapore della crema fresca alla quale rassomigliava, la bocca infantile quello delle fragole. Sotto la massa dei capelli color di notte avvolti in soavi ondulazioni, gli occhi verdi albeggiavano, immoti come quelli delle estatue e, com'essi, un po' crudeli. Procedeva lenta, facendo roteare intorno a sé l'ampia gonna bianca e recava nella persona la pacatezza, l'invincibilità della donna di sicura bellezza (LAMPEDUSA, 1982, p. 52-53).

Dopo l'arrivo disastroso di suo padre, la giovane entra in maniera trionfale nel palazzo, incantando tutti con la sua bellezza. Per Gioia (2014, p.52), Angelica rappresenta la giovane classe sociale insorgente; ha una bellezza animalesca perché s'assomiglia a una bestia piena di vitalità e salute. È opportuno evidenziare che la giovane è del tutto consapevole della forza di sua bellezza, che la rende superiore a tutte le altre donne.

Visconti seppe anche ritrattare il debutto esuberante di Angelica nel palazzo dei Salina, soffermando la macchina da presa sull'entrata lenta di Angelica e sugli sguardi degli altri personaggi che miravano la giovane. La macchina da presa s'avvicina al viso di Angelica e, in seguito, incornicia il suo corpo. Si osserva così che il regista, attraverso le immagini in movimento, cercò di tradurre la descrizione fisica del personaggio fatta dal Lampedusa.

Oltre il narratore, lo spazio e il tempo costituiscono degli elementi importanti nell'articolazione e nello svolgimento del materiale narrativo sia nel cinema che nel testo letterario. Comunque, questi elementi vengono percepiti in maniera diversa nelle opere narrative e filmiche. Pellegrini et al. affermano che tutte le forme narrative, siano letterarie che visive: 
...sono diretta o indirettamente articolate in sequenze temporali, non importa se sono lineari, troncate, invertite o interpolate. La differenza tra la letteratura e il cinema, in questo caso, consiste nel fatto che le sequenze sono costruite con parole nella prima area artistica, e, nella seconda, con le immagini (2003, p.18).

Mentre nella letteratura la questione del tempo può essere descritta con precisione nel corso della lettura, come ad esempio quando si indica, con poche parole, che giorni, mesi, anni sono passati; nel cinema, questa percezione non è così precisa, nonostante la sensazione del tempo che fluisce sia verificata attraverso le immagini in movimento. Nel romanzo in evidenza, $\mathrm{i}$ capitoli iniziano con il mese e l'anno degli avvenimenti, permettendo così al lettore di situarsi nel tempo nel corso della lettura. Il lettore sa esattamente quando inizia la storia, maggio del 1860, e quando essa finisce, maggio del 1910.

Altri indicatori temporali possono ancora essere trovati nel romanzo come ad esempio la durata del viaggio fatto dalla famiglia Salina a Donnafugata: "Il viaggio era durato tre giorni ed era stato orrendo" (LAMPEDUSA, 1982, p. 39). Tutta la famiglia ed i servitori si spostano per le strade calde per passare le vacanze d'estate nella piccola città in cui la famiglia Salina era venerata dagli abitanti.

Nella narrativa filmica, lo spettatore prende atto dell'anno in cui è ambientata la storia attraverso gli avvenimenti storici, in questo caso specifico, l'unificazione italiana avvenuta tra il 1860 e il 1861. La percezione del trascorrere del tempo si verifica attraverso lo spazio che cambia, come ad esempio, quando viene mostrata la famiglia dormendo in un albergo durante il percorso verso Donnafugata. La macchina da presa mette a fuoco le pareti sporche della stanza in cui i membri della famiglia si riposano, mostrandoci lo scomodo di tutti e la decadenza che comincia già ad instaurarsi. Man mano che cambia lo spazio, si percepisce che il tempo è passato, rivelando così che il tempo nel film è, in linea generale, relativo a uno spazio. Questo perché nel 
film il tempo e lo spazio sono inseparabili, poiché i rapporti temporali acquisiscono un aspetto quasi spaziale allo stesso tempo che lo spazio acquisisce delle caratteristiche temporali.

Nel film, l'aspetto temporale si collega allo spazio e la sua rappresentazione, diversamente dal romanzo, è più visibile, dato che nell'opera letteraria ci vogliono molte parole per indicare un'immagine che nel film viene percepita istantaneamente. Nel film, la spazializzazione dell'elemento temporale produce alterazioni profonde nelle maniere di percepire lo spazio e nella maniera di rappresentarlo. A questo proposito, Hauser dice che

Lo spazio perde il suo carattere statico, la sua inerte passività per farsi dinamico; nasce, per così dire, davanti ai nostri occhi. Ė fluido, illimitato, aperto, un elemento che ha la sua storia, i suoi momenti, le sue tappe, $i$ suoi stadi irripetibili." (2003, p. 971).

Perciò Pellegrini et al. adottano i termini invisibili e visibili, per far riferimento, rispettivamente, al tempo e allo spazio: "il tempo, che è invisibile, viene colmato con lo spazio occupato da una sequenza di immagini visibili; si mescolano, così, il visibile e l'invisibile" (2003, p18).

Gaudreault e Jost, in uno studio sulla narrativa cinematografica, sottolineano che lo spazio ha più prestigio del tempo dovuto al carattere immaginifico del film, cioè, lo spazio viene prima del tempo:

E, dato che il fotogramma viene prima della successione dei fotogrammi,
la temporalità nel cinema deve effettivamente basarsi sullo spazio per
arrivare a iscriversi nel centro della narrativa. Il tempo non esiste prima
che si faccia il passaggio tra un primo fotogramma (che è già uno spazio)
e un secondo (che è anche uno spazio) (2009, p. 105).

Dall'altra parte, il narratore del romanzo deve fare una specie di discriminazione, perché esso non può descrivere allo stesso tempo il quadro spaziale e l'azione in cui essa avviene. Così, egli deve scegliere quello che dovrà narrare, eliminando, molte volte delle informazioni spaziali considerate "decorative" e non utili alla narrativa. Grazie alla sua capacità di esprimere tutto, la lingua non potrà mai rappresentare tutti i rapporti di ordine spaziale e, 
perciò, una narrativa scritturale non riuscirà a ritrattare simultaneamente $\mathrm{i}$ parametri spaziali e temporali con cui essa viene confrontata. Ne Il Gattopardo, il paesaggio viene spesso descritto rivelandoci il suo rapporto con la storia:

Il giardino dormiva sprofondato nell'ombra, sotto; nell'aria inerte gli alberi sembravano di piombo fuso; dal campanile incombente giungeva il sibilo fiabesco dei gufi. Il cielo era sgombro di nuvole; quelle che avevano salutato a sera se ne erano andate chissà dove (...) Le stelle apparivano torbide e i loro raggi faticavano a penetrare la coltre di afa (LAMPEDUSA, 1982, p. 57).

Nella narrativa filmica, per contro, lo spazio è sempre presente perché le informazioni relative all'aspetto spaziale sono fornite in abbondanza dalla macchina da presa. Nel cinema, c'è la presentazione simultanea di diversi elementi informazionali e non solo una determinata informazione spaziale. Nel film di Visconti, è interessante osservare che lo spazio rappresenta un elemento importante, poiché la natura viene catturata in diverse ambientazioni. Nella scena in cui Tancredi saluta la famiglia Salina e parte nella sua carrozza per i combattimenti, tutti accorrono sulla terrazza e dal balcone contemplano la partenza del giovane attraverso la vasta proprietà della famiglia. In questa stessa scena, si osserva una fusione tra i personaggi statici e il paesaggio, come se fossero dei veri e propri dipinti.

Si osserva così che, per il cinema, è molto difficile concentrarsi solo nell'azione e non invece nel quadro spaziale in cui essa si svolge. Oltre a ciò, nel film, lo spazio naturale acquisisce un'importanza vitale quando mette in relazione il suo carattere immutabile alla Sicilia. Nella scena in cui il principe e don Ciccio escono per cacciare nelle prime ore del giorno, lo spettatore visualizza gli aspetti geografici del piccolo villaggio in cui si rivela un aspetto selvaggio ed aspro del campo. Lo spettatore prende conoscenza di questo spazio che si rivela attraverso lo spostamento dei due uomini nelle terre aride e improduttive. È in questo posto che don Fabrizio raccoglie le informazioni 
su quello che era avvenuto a Donnafugata negli ultimi mesi e, allo stesso tempo, la natura si presenta davanti agli occhi degli spettatori.

Come accennato prima, la morte è un elemento presente nelle due narrative. Nel film, tuttavia, Visconti decide di escludere il capitolo che narra la morte del protagonista e di dare uno spazio maggiore al ballo offerto dalla famiglia Ponteleone. Mentre il romanzo si conclude con le sorelle Salina già invecchiate, il regista conclude il film con il ballo, che occupa un terzo del film. A proposito della dilatazione del tempo che occupa questa festa, il regista stesso spiega:

La verità è che quella scena era necessaria alla struttura interna del film proprio nella sua apparente sproporzione. Sono 40 minuti che ne rappresentano infinitamente di più (...) Il principe, in quei 40 minuti, invecchia esattamente di quei vent'anni che separavano nel libro il sesto dal settimo capitolo" (ANILE; GIANNICE, 2013, p. 210).

Nel libro, il ballo occupa il sesto capitolo e il lettore viene informato della data della sua realizzazione, cioè novembre 1892. Nel capitolo seguente invece, vent'anni sono già passati e si descrive la morte del principe Salina in una camera d'albergo. Per il regista, che decise di rimuovere dal film la scena della morte del protagonista, era necessario farci allusione sottolineando in tutta la sequenza del ballo lo scorrere del tempo per il principe. Lo spettatore assiste alle scene in cui il protagonista, inizialmente, dà segni di disagio e stanchezza e progressivamente questa scomodità si trasforma in malinconia. La macchina da presa mette in evidenza un grande contrasto tra i giovani che si divertono e sembrano ignorare quello che accadrà e un individuo esaurito fisicamente che osserva tutti con uno sguardo di compassione. Il principe vaga solitario per i saloni del palazzo e i segni fisici della sua stanchezza vengono evidenziati dalle scene in cui frequentemente si deterge il sudore del viso con un fazzoletto e dice a un conoscente che non si sente bene. Il rapporto con la morte diventa più evidente quando il personaggio va alla biblioteca e osserva attentamente il dipinto La morte del Giusto, di Greuze. In questo momento, si 
vede in quel quadro come se stesse davanti a uno specchio, contemplando, così, la propria morte. Come afferma il regista stesso, era necessario trasmettere allo spettatore la percezione che il tempo era passato per il principe e che il suo declino cominciava.

Sia nel romanzo che nel film, si può stabilire un rapporto tra la conclusione del ballo e il declino di un'epoca. Lampedusa descrive la fine del ballo in modo molto preciso: "I volti delle signore erano lividi, gli abiti sgualciti, gli aliti pesanti (...) Nella sala del buffet, vuota, vi erano soltanto piatti smantellati bicchieri con un dito divino..." (LAMPEDUSA, 1982, p. 160). Visconti tesse anche questa descrizione attraverso la cinepresa che si muove entro la scena, permettendo allo spettatore di intravedere l'albeggiare del giorno e gli invitati, stanchi e sudati, salutando gli anfitrioni in uno spazio che, all'inizio, veniva segnalato dal lusso e dall'abbondanza e, adesso, totalmente in disordine, con piatti e bicchieri vuoti sparpagliati sulla tavola. Inoltre, il regista aggiunge un altro elemento che ci permette di intravedere l'uscita di scena dell'aristocrazia per dare spazio alla nuova borghesia. Si tratta del dialogo tra Angelica e Concetta, figlia del principe, che si dice stanca di frequentare tanti balli mentre quella dice che si sente felice di partecipare alle feste organizzate. In altre parole, la classe aristocratica esce di scena per lasciare il posto alla nuova classe che verrà istituita. Il ballo, allora, può essere interpretato come metafora del tramonto dell'aristocrazia e la nascita della borghesia.

È durante il ballo che Angelica viene presentata all'aristocrazia come fidanzata di Tancredi. La partecipazione della giovane è il coronamento dell'interessata storia d'amore con Tancredi e, allo stesso tempo, il trionfo della nuova classe dirigente: "...Angelica che invita Don Fabrizio a farla danzare è la borghesia che seduce l'aristocrazia un attimo prima di prenderne il posto..." (ANILE; GIANNICE, 2013, p. 209). Il principe, che dall'entrata di Angelica nella di lui casa si era sentito sedotto dalla bellezza e dall'esuberanza della giovane, favorisce il matrimonio del nipote Tancredi con 
la bella ragazza. Oltre ad essere bella, Angela ha altre qualità che la fa diventare la scelta giusta come sposa: ha un'ingente fortuna economica e una forte presenza; rivelandosi pertanto la donna ideale per un giovane ambizioso come Tancredi.

Dopo aver chiesto a Tancredi di provvedere una carrozza per la sua famiglia, il principe torna a casa a piedi davanti una città che si sveglia. Ancora una volta la morte si farà presente nei cammini del protagonista perché, mentre fa il suo percorso verso casa, apparisce un prete che sta portando l'estrema unzione a un malato. La macchina da presa inquadra il principe che s'inginocchia al passaggio del Viatico e, in seguito, dopo aver visto il dolore e la sofferenza, si alza, osserva i primi raggi di sole che spuntano e cammina tra le viuzze, allontanandosi, di spalle, dalla cinepresa.

Mentre il film finisce con il rientro del principe a casa, nel romanzo, nel capitolo settimo, viene descritta la sua morte, lentamente già annunciata in diversi momenti della storia, in una stanza di un albergo. Egli osserva i figli ed i nipoti che circondano il suo letto e si rende conto che nessuno si somiglia a lui, cioè, in quel momento viene estinta la stirpe, dei "Gattopardi". Dopo, passati tre decenni, l'opera si conclude con le sorelle Salina cercando ancora di mantenere il prestigio della famiglia attraverso le reliquie religiose che riuscirono a riunire per parecchie decadi. Lampedusa pare, così, rivelare un ultimo vestigio della vita di una famiglia tradizionale che visse il suo apogeo e assistette alla sua sostituzione con un'altra classe sociale.

Si verificò allora che la traduzione cinematografica è un intervento in cui occorre una trasformazione audiovisiva di un testo letterario, mettendo in evidenza un dato tipo di interpretazione e quindi presentando una rilettura del testo letterario. Come osservato prima, Visconti si avvicinò molto all'opera di partenza, rifacendo i dialoghi, le situazioni e l'ambiente in cui le scene si verificarono, ma presentò un'altra lettura nella conclusione del suo film 
quando ne escluse alcuni capitoli del libro, dando maggior rilievo a un altro specifico.

\section{Riferimenti bibliografici}

ANILE, Alberto; GIANNICE, Maria Gabriella. Operazione Gattopardo. Genova: Feltrinelli, 2013.

AUMONT et al. A estética do filme. Campinas: Papirus, 2013.

BALDI et al. Testi e storia della Letteratura. Dal dopoguerra ai giomi nostri. Vol. G. Torino: Paravia, 2011.

BETTON, Gérard. Estética do cinema. São Paulo: Martins Fontes, 1987.

COUTINHO, Evaldo. A imagem autônoma. São Paulo: Editora Perspectiva, 1996.

ECO, Umberto. Quase a mesma coisa. Rio de Janeiro: BestBolso, 2011.

GAUDREAULT, Andre. JOST, François. A narrativa cinematográfica. Brasília: EditoraUniversidade de Brasília, 2009.

GIOIA, Edvige. Il mondo decadente del Gattopardo. Roma: Aracne editrice, 2014.

HAUSER, Arnold. História social da arte e da literatura. São Paulo: Martins Fontes, 2003.

LAMPEDUSA, Giuseppe. Il Gattopardo. Milano: Feltrinelli, 1982.

MOSCARIELLO, Angelo. Cinema e/o letteratura. Bologna: Pitagora Editrice, 1981.

PELLEGRINI, Tânia et al. Literatura, cinema e televisão. São Paulo: Ed. Senac São Paulo, 2003.

PLAZA, Julio. Traducão Intersemiótica. São Paulo: Ed. Perspectiva, 2010. 
A tradução cinematográfica de /l Gattopardo, de Giuseppe Tomasi di Lampedusa Marinês Lima Cardoso

\section{Referenze filmiche}

Il Gattopardo. Direção: Luchino Visconti. Produção: Goffredo Lombardo. Roteiro: Suso Cecchi D’Amico, Luchino Visconti, Pasquale Festa Campanile, Enrico Medioli. Versátil, 1963. 185 min, colorido. 